\title{
The Age of the Earth
}

The chessboard is the world, the pieces are the phenomena of the universe, the rules of the game are what we call the laws of nature. The player on the other side is hidden from us. We know that his play is always fair, just, and patient. But also we know, to our cost, that he never overlooks a mistake, or makes the smallest allowance for ignorance.

T. H. HUXLEY, 1868

A Liberal Education 
